\title{
Global point dissipativity of neural networks with mixed time-varying delays
}

\author{
Jinde $\mathrm{Cao}^{\mathrm{a})}$ and Kun Yuan \\ Department of Mathematics, Southeast University, Nanjing 210096, People's Republic of China \\ Daniel W. C. Ho ${ }^{\text {b) }}$ \\ Department of Mathematics, City University of Hong Kong, Hong Kong \\ James Lam \\ Department of Mechanical Engineering, University of Hong Kong, Hong Kong
}

(Received 14 July 2005; accepted 30 September 2005; published online 12 January 2006)

\begin{abstract}
By employing the Lyapunov method and some inequality techniques, the global point dissipativity is studied for neural networks with both discrete time-varying delays and distributed time-varying delays. Simple sufficient conditions are given for checking the global point dissipativity of neural networks with mixed time-varying delays. The proposed linear matrix inequality approach is computationally efficient as it can be solved numerically using standard commercial software. Illustrated examples are given to show the usefulness of the results in comparison with some existing results. () 2006 American Institute of Physics. [DOI: 10.1063/1.2126940]
\end{abstract}

It is well known that the stability problem is central to the analysis of a dynamic system, especially the stability of an equilibrium point that has captured the attention of researchers. Nevertheless, from a practical point of view, it is not always the case that every dynamic system has its orbits approach a single equilibrium point. It is possible that there is no equilibrium point in some situations. Therefore, the concept such as point dissipativity has been introduced and investigated. The concept of point dissipativity generalizes the idea of a Lyapunov stability and has found application in diverse areas such as stability theory, chaos and synchronization theory, system norm estimation, and robust control. To the best of our knowledge, so far the point dissipativity problem for general neural networks with both discrete and distributed delays has received little research attention, mainly due to the mathematical difficulties in dealing with discrete and distributed delays simultaneously. Hence, it is our intention in this paper to tackle such an important yet challenging problem. In this paper, simple sufficient conditions are given for checking the global point dissipativity of neural networks with mixed time-varying delays by employing the Lyapunov method and some inequality techniques.

\section{INTRODUCTION}

In recent years, neural networks have been extensively investigated and many successful applications in different areas were found. Such applications depend heavily on the dynamic behavior of the networks. The dynamic behaviors of various neural networks, such as the stability, the attraction, and the oscillation, are popular research topics that have drawn much attention from mathematics, physicists, and

\footnotetext{
${ }^{a)}$ Electronic mail: jdcao@seu.edu.cn

${ }^{b)}$ Electronic mail: madaniel@cityu.edu.hk
}

computer sciences, and a large amount of results are available in the literature (see Refs. 1-20 and references therein). In Refs. 1 and 2 several sufficient conditions are presented for complete stability of delayed cellular neural networks with positive cell linking and dominant templates. In Refs. 16-19 several sufficient conditions have been derived for absolute stability of neural networks. In Refs. 3-15 and 31 the conditions for the existence of an equilibrium point, and the global stability of various neural networks with delay are investigated yielding results of significant generality which are expected to be useful in network design. Various sufficient conditions, either delay dependent or delay independent, have been proposed to guarantee global asymptotic or exponential stability for neural networks. However, in these recent publications, only discrete time delays have been treated.

Another type of time delay, namely, distributed delays, have begun to receive research attention. The main reason is that a neural network usually has a spatial nature due to the presence of an amount of parallel pathways of a variety of axon sizes and lengths, therefore, it is desirable to model them by introducing continuously distributed delays over a certain duration of time, such that the distant past has less influence compared to the recent behavior of the state. ${ }^{21}$ Therefore, when modeling neural networks, both discrete and distributed time delays should be taken into account. ${ }^{22}$ Very recently, many results have been reported on the stability issue for various neural networks with distributed time delays. ${ }^{23-26}$ As is well known, the stability problem is central to the analysis of a dynamic system where various types of stability of an equilibrium point have captured the attention of researchers. Nevertheless, from a practical point of view, it is not always the case that every neural network has its orbits approach a single equilibrium point. It is possible that there is no equilibrium point in some situations. Therefore, the concept such as point dissipativity has been introduced ${ }^{34}$ 
and investigated in Refs. 27-29. The concept of point dissipativity generalizes the idea of a Lyapunov stability and has found applications in diverse areas such as stability theory, chaos and synchronization theory, system norm estimation, and robust control. ${ }^{28,29}$ To the best of our knowledge, so far the point dissipativity problem for general neural networks with both discrete and distributed delays has received little research attention, mainly due to the mathematical difficulties in dealing with discrete and distributed delays simultaneously. Hence, it is our intention in this paper to tackle such an important yet challenging problem. Moreover, in practice, time-varying delay in neural networks occurs commonly in most designs. Thus, the study of neural networks with timevarying delays is more important in practice than those with constant delays.

Motivated by the above discussion, the aims of this paper are to study the global point dissipativity of neural networks with both discrete time-varying delays and distributed time-varying delays. For the different classes of activation functions, we derive several new criteria for checking the global point dissipativity of the considered model by using different bounding techniques and Lyapunov functions or functionals.

The paper is organized as follows: In Sec. II the model formulation and some preliminaries are given. The main results are stated in Sec. III. Illustrated examples are given to demonstrate the effectiveness of the proposed results in Sec. IV. Finally, concluding remarks are made in Sec. V.

Notation: Throughout this paper, for real symmetric matrices $X$ and $Y$, the notation $X \geqslant Y$ (respectively, $X>Y$ ) means that the matrix $X-Y$ is positive semidefinite (respectively, positive definite). The superscript " $T$ " represents the transpose. The notation $\|x\|_{r}$ denotes a vector norm defined by $\|x\|_{r}=\left(\sum_{i=1}^{n} x_{i}^{r}\right)^{1 / r}(r \geqslant 1)$ when $x$ is a vector. For a matrix $A,\|A\|$ denotes the spectral norm defined by $\|A\|=\left[\lambda_{\max }\left(A^{T} A\right)\right]^{1 / 2}$. Matrix dimensions, if not explicitly stated, are assumed to be compatible for algebraic operations. For sets $\mathcal{A}$ and $\mathcal{B}$, the set difference $\mathcal{A} \backslash \mathcal{B}$ is defined as $\{x \mid x \in \mathcal{A}$ and $x \notin \mathcal{B}\}$.

\section{MODEL FORMULATION AND PRELIMINARIES}

Consider the following neural networks with both timevarying discrete delays and distributed time-varying delays:

$$
\begin{aligned}
\frac{d x(t)}{d t}= & -D x(t)+A f[x(t)]+B f\left\{x\left[t-\tau_{1}(t)\right]\right\} \\
& +C \int_{t-\tau_{2}(t)}^{t} f[x(s)] d s+u,
\end{aligned}
$$

or

$$
\begin{aligned}
\frac{d x_{i}(t)}{d t}= & -d_{i} x_{i}(t)+\sum_{j=1}^{n} a_{i j} f_{j}\left[x_{j}(t)\right]+\sum_{j=1}^{n} b_{i j} f_{j}\left\{x_{j}\left[t-\tau_{1}(t)\right]\right\} \\
& +\sum_{j=1}^{n} c_{i j} \int_{t-\tau_{2}(t)}^{t} f_{j}\left[x_{j}(s)\right] d s+u_{i}
\end{aligned}
$$

where $x(t)=\left[x_{1}(t), x_{2}(t), \ldots, x_{n}(t)\right]^{T} \in \mathbb{R}^{n}$ is the state vector associated with the neurons, $D=\operatorname{diag}\left(d_{1}, d_{2}, \ldots, d_{n}\right)>0 ; A$ $=\left(a_{i j}\right)_{n \times n}, B=\left(b_{i j}\right)_{n \times n}$, and $C=\left(c_{i j}\right)_{n \times n}$ denote the connection weights matrix, the connection weights matrix with discrete delays, and the connection weights with distributed delays, respectively; $u=\left(u_{1}, u_{2}, \ldots, u_{n}\right)^{T} \in \mathbb{R}^{n}$ is a constant external input vector; $\tau_{1}(t)$ and $\tau_{2}(t)$ are discrete and distributed timevarying delays, respectively; and $f_{j}$ is a real activation function,

$$
\begin{gathered}
f[x(t)]=\left\{f_{1}\left[x_{1}(t)\right], f_{2}\left[x_{2}(t)\right], \ldots, f_{n}\left[x_{n}(t)\right]\right\}^{T} \in \mathbb{R}^{n}, \\
f\left\{x\left[t-\tau_{1}(t)\right]\right\}=\left(f_{1}\left\{x_{1}\left[t-\tau_{1}(t)\right]\right\}, f_{2}\left\{x_{2}\left[t-\tau_{1}(t)\right]\right\}, \ldots,\right. \\
\left.f_{n}\left\{x_{n}\left[t-\tau_{1}(t)\right]\right\}\right)^{T} \in \mathbb{R}^{n} .
\end{gathered}
$$

Throughout this paper, the time-varying delays are assumed to satisfy the following assumption.

Assumption A: The time-varying delays $\tau_{1}(t)$ and $\tau_{2}(t)$ are positive, bounded, and differentiable functions with 0 $<\tau_{1}(t) \leqslant \tau_{1}, \quad 0<\tau_{2}(t) \leqslant \tau_{2}$ and $0 \leqslant \tau_{1}^{\prime}(t) \leqslant \eta_{1}<1,0 \leqslant \tau_{2}^{\prime}(t)$ $\leqslant \eta_{2}<1$.

Next, we give the definitions of several classes of activation functions:

(i) A function $g(x)=\left[g_{1}\left(x_{1}\right), g_{2}\left(x_{2}\right), \ldots, g_{n}\left(x_{n}\right)\right]$ is of class $\mathcal{L}$ if for $\forall x_{i} \in \mathbb{R}, x_{i} \neq 0$, it satisfies $0<g_{i}\left(x_{i}\right) / x_{i} \leqslant l_{i}$, and $g_{i}(0)=0, i=1,2, \ldots, n$;

A function $g(x)=\left[g_{1}\left(x_{1}\right), g_{2}\left(x_{2}\right), \ldots, g_{n}\left(x_{n}\right)\right]$ is of class $\mathcal{L}^{*}$ if for $\forall x_{i} \in \mathbb{R}, x_{i} \neq 0$, it satisfies $0 \leqslant g_{i}\left(x_{i}\right) / x_{i} \leqslant l_{i}, l_{i}>0$, and $g_{i}(0)=0, i=1,2, \ldots, n$;

(ii) A function $g(x)=\left[g_{1}\left(x_{1}\right), g_{2}\left(x_{2}\right), \ldots, g_{n}\left(x_{n}\right)\right]$ is of class $\mathcal{G}$ if it satisfies $0<D^{+} g_{i}\left(x_{i}\right)<+\infty$, and $g_{i}(0)=0, i$ $=1,2, \ldots, n$;

A function $g(x)=\left[g_{1}\left(x_{1}\right), g_{2}\left(x_{2}\right), \ldots, g_{n}\left(x_{n}\right)\right]$ is of class $\mathcal{G}^{*}$ if it satisfies $0 \leqslant D^{+} g_{i}\left(x_{i}\right)<+\infty$, and $g_{i}(0)=0, i=1,2, \ldots, n$;

(iii) A function $g(x)=\left[g_{1}\left(x_{1}\right), g_{2}\left(x_{2}\right), \ldots, g_{n}\left(x_{n}\right)\right]$ is of class $\mathcal{B}$ if it satisfies $\left|g_{i}\left(x_{i}\right)\right| \leqslant h_{i}, i=1,2, \ldots, n$.

Similar to Ref. 27 we also give the following definitions.

Definition 1: The neural network model (1) is said to be a globally point dissipative, if there exists a compact set, $\mathcal{S} \subset \mathbb{R}^{n}$, such that $\forall x_{0} \in \mathbb{R}^{n}, \exists T\left(x_{0}\right)>0$, when $t>t_{0}+T\left(x_{0}\right)$, $x\left(t, t_{0}, x_{0}\right) \subseteq \mathcal{S}$, where $x\left(t, t_{0}, x_{0}\right)$ denotes the solution of $(1)$ from the initial state $x_{0}$ and initial time $t_{0}$. In this case, $\mathcal{S}$ is called a globally attractive set. A set $\mathcal{S}$ is called a positive invariant, if $\forall x_{0} \in \mathcal{S}$ implies $x\left(t, t_{0}, x_{0}\right) \subseteq \mathcal{S}$ for $t \geqslant t_{0}$.

Definition 2: Let $\mathcal{S}$ be a globally attractive set of neural network models (1). The neural network model (1) is said to be a globally exponentially dissipative system, if there exists a compact $\mathcal{S}^{*} \subset \mathcal{S}$ in $\mathbb{R}^{n}$ such that $\forall x_{0} \in \mathbb{R}^{n} \backslash \mathcal{S}^{*}$, there exist a constant $M\left(x_{0}\right)$ and $\alpha>0$ such that

$$
\inf _{x_{0} \in \mathbb{R}^{n} \mathcal{S}^{*}}\left\{\left\|x\left(t, t_{0}, x_{0}\right)-\widetilde{x}\right\|_{r} \mid \widetilde{x} \in \mathcal{S}^{*}\right\} \leqslant M\left(x_{0}\right) \exp \left\{-\alpha\left(t-t_{0}\right)\right\} .
$$

The set $\mathcal{S}^{*}$ is said to be a globally exponentially attractive.

To obtain our main results, we need the following lemmas.

Lemma 1 (Ref. 30): Given any real matrices $X, Y, P$ $>0$ of appropriate dimensions, the following inequality holds:

$$
X^{T} Y+Y^{T} X \leqslant X^{T} P X+Y^{T} P^{-1} Y .
$$

Lemma 2 (Schur Complement, Ref. 32): For a given matrix, 


$$
S=\left[\begin{array}{ll}
S_{11} & S_{12} \\
S_{12}^{T} & S_{22}
\end{array}\right]<0
$$

is equivalent to any one of the following conditions:

(i) $S_{22}<0, S_{11}-S_{12} S_{22}^{-1} S_{12}^{T}<0$;

(ii) $S_{11}<0, S_{22}-S_{12}^{T} S_{11}^{-1} S_{12}<0$.

Lemma 3 (Jensen's Inequality, Ref. 33): For any constant matrix, $V \in \mathbb{R}^{m \times m}, V>0$, scalar $0<r(t)<r$, vector function $\nu:[0, r] \rightarrow \mathbb{R}^{m}$ such that the integrations concerned are well defined, then

$r(t) \int_{0}^{r(t)} \nu^{T}(s) V \nu(s) d s \geqslant\left(\int_{0}^{r(t)} \nu(s) d s\right)^{T} V\left(\int_{0}^{r(t)} \nu(s) d s\right)$.

Consider the following equations:

$$
\frac{d x_{i}(t)}{d t}=f_{i}\left(t, x_{t}\right), \quad i=1,2, \ldots, n,
$$

where $f_{i}(t, \phi):[0,+\infty) \times C[-\tau, 0] \rightarrow \mathrm{R}$ is continuous with respect to $(t, \phi)$.

Lemma 4: Let $V \in C\left(\mathbb{R}^{n}, \mathbb{R}\right)$ satisfy $V(x) \rightarrow \infty$ as $\|x\|_{r}$ $\rightarrow \infty$. Assume there exists a set $E=\left\{x \in \mathbb{R}^{n} \mid V(x) \leqslant K\right\}$ for some $K>0$ such that the Dini derivative $D^{+} V(x)$ of $V(x)$ along with solution $x(t)$ of (4) satisfies $\left.D^{+} V(x)\right|_{(4)}<0$ as long as $x(t) \in \mathbb{R}^{n} \backslash E$. Then (4) is point dissipative in $\mathbb{R}^{n}$.

Proof: Define $E_{1}=\left\{x \in \mathbb{R}^{n}: V(x)<K+1\right\}$. Then $E \subset E_{1}$ and $E_{1}$ is bounded. Furthermore, $\left.D^{+} V(x)\right|_{(1)}<0$ for $x$ $\in \mathbb{R}^{n} \backslash E_{1}$. We will show that for any $x_{0} \in \mathbb{R}^{n}$, there is a $T$ $\geqslant t_{0}$ such that $x\left(t, t_{0}, x_{0}\right) \in E_{1}$ for $t \geqslant T$, as long as $x$ is continuous in $\mathbb{R}^{n}$. This is obviously true if $x_{0} \in E_{1}$. So we consider the case that $x_{0} \in \mathbb{R}^{n} \backslash E_{1}$. Let $E_{2}=\left\{x \in \mathbb{R}^{n}: K+1 \leqslant V(x)\right.$ $\left.\leqslant V\left(x_{0}\right)\right\}$. If the conclusion is not true, then $x\left(t, t_{0}, x_{0}\right) \in E_{2}$ for $t \geqslant t_{0}$. Thus along the solution, $\left.D^{+} V(x)\right|_{(4)} \leqslant l$, where $l$ $=\max \left\{D^{+} V(x) \mid x \in E_{2}\right\}<0$. Hence $V[x(t)] \leqslant l\left(t-t_{0}\right) \rightarrow-\infty$ as $t \rightarrow \infty$. This contradiction completes the proof.

Remark 1: The considered model (1) and the model given in Ref. 29 are the special cases of (4). The proof of Lemma 5 is a minor modification of the proof of Lemma 1.1 in Ref. 29.

\section{MAIN RESULTS}

\section{A. Results using Lyapunov-Krasovskii functionals}

In this section, conditions are given for global point dissipativity of (1) by constructing appropriate LyapunovKrasovskii functionals and employing different bounding techniques.

Theorem 1: Let assumption A be satisfied. Neural network model (1) is globally point dissipative, if there exists matrices $Q_{1}>0, Q_{2}>0$, and diagonal $K>0$ such that the following linear matrix inequality (LMI) holds:
$\left[\begin{array}{ccc}K A+A^{T} K+Q_{1}+Q_{2} & K B & \tau_{2} K C \\ B^{T} K & -\left(1-\eta_{1}\right) Q_{1} & 0 \\ \tau_{2} C^{T} K & 0 & -\left(1-\eta_{2}\right) Q_{2}\end{array}\right]<0$.

Moreover,

(i) if $f(x) \in \mathcal{L}$, the set $S_{1}=\left\{x|| f_{i}\left(x_{i}\right)\left|\leqslant l_{i}\right| u_{i} \mid / d_{i}, i\right.$ $=1,2, \ldots, n\}$ is positively invariant and globally attractive;

(ii) if $f(x) \in \mathcal{L}^{*}$, the set $S_{2}=\left\{x|| f_{i}\left(x_{i}\right)\left|\leqslant \varepsilon l_{i}\right| u_{i} \mid / d_{i}, i\right.$ $=1,2, \ldots, n\}$ where $\varepsilon>1$, is positively invariant and globally attractive;

(iii) if $f(x) \in \mathcal{G}$, the set $\bar{S}_{1}=\left\{x|| x_{i}|\leqslant| u_{i} \mid / d_{i}, i=1,2, \ldots, n\right\}$ is positively invariant and globally attractive;

(iv) if $f(x) \in \mathcal{G}^{*}$, the set $\bar{S}_{2}=\left\{x|| x_{i}|\leqslant \varepsilon| u_{i} \mid / d_{i}, i\right.$ $=1,2, \ldots, n\}$ where $\varepsilon>1$, is positively invariant and globally attractive.

Proof: First, denote

$x_{t}=x(t+\theta), \quad-\max \left\{\tau_{1}, \tau_{2}\right\} \leqslant \theta \leqslant 0$.

(i) Consider the following positive radially unbounded Lyapunov-Krasovskii functional candidate:

$$
\begin{aligned}
V\left(x_{t}\right)= & 2 \sum_{i=1}^{n} k_{i} \int_{0}^{x_{i}} f_{i}(s) d s+\int_{t-\tau_{1}(t)}^{t} f^{T}[x(s)] Q_{1} f[x(s)] d s \\
& +\tau_{2}^{-1} \int_{t-\tau_{2}(t)}^{t} \int_{\theta}^{t} f^{T}[x(s)] Q_{2} f[x(s)] d s d \theta,
\end{aligned}
$$

where $K=\operatorname{diag}\left(k_{1}, k_{2}, \ldots, k_{n}\right)$. By calculating the derivative along the positive half trajectory of (1), we have

$$
\begin{aligned}
\frac{d V}{d t}= & 2 \sum_{i=1}^{n} k_{i} f_{i}\left[x_{i}(t)\right]\left[-d_{i} x_{i}(t)+u_{i}\right]+f^{T}[x(t)] \\
& \times\left(K A+A^{T} K\right) f[x(t)]+2 f^{T}[x(t)] K B f\left\{x\left[t-\tau_{1}(t)\right]\right\} \\
& +2 f^{T}[x(t)] K C \int_{t-\tau_{2}(t)}^{t} f[x(s)] d s+f^{T}[x(t)] Q_{1} f[x(t)] \\
& -\left[1-\tau_{1}^{\prime}(t)\right] f^{T}\left\{x\left[t-\tau_{1}(t)\right]\right\} Q_{1} f\left\{x\left[t-\tau_{1}(t)\right]\right\} \\
& -\tau_{2}^{-1}\left[1-\tau_{2}^{\prime}(t)\right] \int_{t-\tau_{2}(t)}^{t} f^{T}[x(s)] Q_{2} f[x(s)] d s \\
& +\tau_{2}^{-1} \tau_{2}(t) f^{T}[x(t)] Q_{2} f[x(t)] .
\end{aligned}
$$

From the Jensen inequality, we have

$$
\begin{aligned}
& -\int_{t-\tau_{2}(t)}^{t} f^{T}[x(s)] Q_{2} f[x(s)] d s \\
& \quad \leqslant-\tau_{2}^{-1}\left(\int_{t-\tau_{2}(t)}^{t} f[x(s)] d s\right)^{T} Q_{2}\left(\int_{t-\tau_{2}(t)}^{t} f[x(s)] d s\right) .
\end{aligned}
$$

By Lemma 1, the following inequalities hold: 


$$
\begin{aligned}
2 f^{T}[x(t)] K C \int_{t-\tau_{2}(t)}^{t} f[x(s)] d s \\
\leqslant \\
\quad \tau_{2}^{-2}\left[1-\tau_{2}^{\prime}(t)\right]\left(\int_{t-\tau_{2}(t)}^{t} f[x(s)] d s\right)^{T} \\
\quad \times Q_{2}\left(\int_{t-\tau_{2}(t)}^{t} f[x(s)] d s\right) \\
\quad+\tau_{2}^{2}\left[1-\tau_{2}^{\prime}(t)\right]^{-1} f^{T}[x(t)] K C Q_{2}^{-1} C^{T} K f[x(t)],
\end{aligned}
$$

$$
\begin{aligned}
2 f^{T}[x(t)] K B f\left\{x\left[t-\tau_{1}(t)\right]\right\} \\
\leqslant \\
\quad\left[1-\tau_{1}^{\prime}(t)\right] f^{T}\left\{x\left[t-\tau_{1}(t)\right]\right\} Q_{1}\left\{x\left[t-\tau_{1}(t)\right]\right\} \\
\quad+\left[1-\tau_{1}^{\prime}(t)\right]^{-1} f^{T}[x(t)] K B Q_{1}^{-1} B^{T} K f[x(t)] .
\end{aligned}
$$

It follows from inequalities (8)-(10) that

$$
\begin{aligned}
\frac{d V}{d t} \leqslant & 2 \sum_{i=1}^{n} k_{i} f_{i}\left[x_{i}(t)\right]\left(-\frac{d_{i}}{l_{i}} f_{i}\left[x_{i}(t)\right]+u_{i}\right)+f^{T}[x(t)] \\
& \times\left[K A+A^{T} K+Q_{1}+Q_{2}+\tau_{2}^{2}\left(1-\eta_{2}\right)^{-1}\right. \\
& \left.\times K C Q_{2}^{-1} C^{T} K+\left(1-\eta_{1}\right)^{-1} K B Q_{1}^{-1} B^{T} K\right] f[x(t)] \\
= & 2 \sum_{i=1}^{n} k_{i} f_{i}\left[x_{i}(t)\right]\left(-\frac{d_{i}}{l_{i}} f_{i}\left[x_{i}(t)\right]+u_{i}\right)+f^{T}[x(t)] \Omega f[x(t)]
\end{aligned}
$$

$<-2 \sum_{i=1}^{n} k_{i} \frac{d_{i}}{l_{i}}\left|f_{i}\left[x_{i}(t)\right]\right|\left(\left|f_{i}\left[x_{i}(t)\right]\right|-\left|u_{i}\right| \frac{l_{i}}{d_{i}}\right)<0$,

when $x \in \mathbb{R}^{n} \backslash S_{1}$,

where $\quad \Omega=K A+A^{T} K+Q_{1}+Q_{2}+\tau_{2}^{2}\left(1-\eta_{2}\right)^{-1} K C Q_{2}^{-1} C^{T} K$ $+\left(1-\eta_{1}\right)^{-1} K B Q_{1}^{-1} B^{T} K$. (11) holds because of the negative definiteness of $\Omega$. By the Schur complement in Lemma 3, $\Omega<0$ if and only if (5) holds. Thus the neural network model (1) is a globally point dissipative system, and the set $S_{1}$ is a positive invariant and globally attractive set as (5) holds.

(ii) For any $\varepsilon>1$, since $\Omega<0$, we can choose a sufficiently small $P=\operatorname{diag}\left(p_{1}, p_{2}, \ldots, p_{n}\right)>0$ such that

$$
\begin{gathered}
{\left[\begin{array}{ccc}
K A+A^{T} K+Q_{1}+Q_{2} & K B & \tau_{2} K C \\
B^{T} K & -\left(1-\eta_{1}\right) Q_{1} & 0 \\
\tau_{2} C^{T} K & 0 & -\left(1-\eta_{2}\right) Q_{2}
\end{array}\right]} \\
+\frac{\varepsilon}{2(\varepsilon-1)}\left[\begin{array}{c}
A^{T} \\
B^{T} \\
\tau_{2} C^{T}
\end{array}\right] P D^{-1}\left[\begin{array}{lll}
A & B & \tau_{2} C
\end{array}\right]<0,
\end{gathered}
$$

or equivalently,

$$
\Omega^{\prime}=\left[\begin{array}{cccc}
-2\left(1-\frac{1}{\varepsilon}\right) P D & P A & P B & \tau_{2} P C \\
A^{T} P & K A+A^{T} K+Q_{1}+Q_{2} & K B & \tau_{2} K C \\
B^{T} P & B^{T} K & -\left(1-\eta_{1}\right) Q_{1} & 0 \\
\tau_{2} C^{T} P & \tau_{2} C^{T} K & 0 & -\left(1-\eta_{2}\right) Q_{2}
\end{array}\right]<0 .
$$

Next, we consider the following Lyapunov-Krasovskii functional:

$$
\widetilde{V}\left(x_{t}\right)=x^{T} P x+V\left(x_{t}\right),
$$

where $V\left(x_{t}\right)$ is defined in (6). By calculating the derivative along the positive half trajectory of (1), we have

$$
\begin{aligned}
\frac{d \tilde{V}}{d t}= & \dot{x}^{T}(t) P x+x^{T}(t) P \dot{x}(t)+\frac{d V}{d t} \\
= & -2 x^{T}\left(1-\frac{1}{\varepsilon}\right) P D x(t)+2 x^{T}(t) P A f[x(t)]+2 x^{T}(t) P B f\left\{x\left[t-\tau_{1}(t)\right]\right\}+2 x^{T}(t) P C \int_{t-\tau_{2}(t)}^{t} f[x(s)] d s \\
& +2 \sum_{i=1}^{n} \frac{1}{\varepsilon} x_{i}(t) p_{i}\left[-d_{i} x_{i}(t)+\varepsilon u_{i}\right]+2 \sum_{i=1}^{n} k_{i} f_{i}\left[x_{i}(t)\right]\left[-d_{i} x_{i}(t)+u_{i}\right]+f^{T}[x(t)]\left(K A+A^{T} K\right) f[x(t)] \\
& +2 f^{T}[x(t)] K B f\left\{x\left[t-\tau_{1}(t)\right]\right\}+2 f^{T}[x(t)] K C \int_{t-\tau_{2}(t)}^{t} f[x(s)] d s+f^{T}[x(t)] Q_{1} f[x(t)] \\
& -\left[1-\tau_{1}^{\prime}(t)\right] f^{T}\left\{x\left[t-\tau_{1}(t)\right]\right\} Q_{1} f\left\{x\left[t-\tau_{1}(t)\right]\right\}-\tau_{2}^{-1}\left[1-\tau_{2}^{\prime}(t)\right] \int_{t-\tau_{2}(t)}^{t} f^{T}[x(s)] Q_{2} f[x(s)] d s+\tau_{2}^{-1} \tau_{2}(t) f^{T}[x(t)] Q_{2} f[x(t)] \\
\leqslant & -2 x^{T}\left(1-\frac{1}{\varepsilon}\right) P D x(t)+2 x^{T}(t) P A f[x(t)]+2 x^{T}(t) P B f\left\{x\left[t-\tau_{1}(t)\right]\right\}+2 x^{T}(t) P C \int_{t-\tau_{2}(t)}^{t} f[x(s)] d s
\end{aligned}
$$




$$
\begin{aligned}
& +2 \sum_{i=1}^{n} \frac{p_{i}}{\varepsilon}\left|x_{i}(t)\right|\left(-\frac{d_{i}}{l_{i}}\left|f_{i}\left[x_{i}(t)\right]\right|+\varepsilon\left|u_{j}\right|\right)+2 \sum_{i=1}^{n} k_{i}\left|f_{i}\left[x_{i}(t)\right]\right|\left(-\frac{d_{i}}{l_{i}}\left|f_{i}\left[x_{i}(t)\right]\right|+\left|u_{i}\right|\right)+f^{T}[x(t)]\left(K A+A^{T} K\right) f[x(t)] \\
& +2 f^{T}[x(t)] K B f\left\{x\left[t-\tau_{1}(t)\right]\right]+2 f^{T}[x(t)] K C \int_{t-\tau_{2}(t)}^{t} f[x(s)] d s+f^{T}[x(t)] Q_{1} f[x(t)] \\
& -\left(1-\eta_{1}\right) f^{T}\left\{x\left[t-\tau_{1}(t)\right]\right\} Q_{1} f\left\{x\left[t-\tau_{1}(t)\right]\right\} \\
& -\tau_{2}^{-2}\left(1-\eta_{2}\right)\left(\int_{t-\tau_{2}(t)}^{t} f[x(s)] d s\right)^{T} Q_{2}\left(\int_{t-\tau_{2}(t)}^{t} f[x(s)] d s\right)+f^{T}[x(t)] Q_{2} f[x(t)] \\
& =2 \sum_{i=1}^{n} \frac{p_{i}}{\varepsilon}\left|x_{i}(t)\right|\left(-\frac{d_{i}}{l_{i}}\left|f_{i}\left[x_{i}(t)\right]\right|+\varepsilon\left|u_{i}\right|\right)+2 \sum_{i=1}^{n} k_{i}\left|f_{i}\left[x_{i}(t)\right]\right|\left(-\frac{d_{i}}{l_{i}}\left|f_{i}\left[x_{i}(t)\right]\right|+\left|u_{i}\right|\right)+\xi^{T}(t) \Omega^{\prime} \xi(t)<0, \quad \text { when } x \in \mathbb{R}^{n} \backslash S_{2},
\end{aligned}
$$

where $\quad \xi^{T}(t)=\left\{x^{T}(t), f^{T}[x(t)], f^{T}\left\{x\left[t-\tau_{1}(t)\right]\right\}, \tau_{2}^{-1} \int_{t-\tau_{2}(t)}^{t}\right.$ $\left.\times f^{T}[x(s)] d s\right\}$. Equation (15) holds because of (13). Hence, when (5) holds, the neural network model (1) is globally point dissipative, and the set $S_{2}$ is positively invariant and globally attractive.

(iii) Consider the Lyapunov-Krasovskii functional candidate as in (6). Similar to (11), we have

$$
\begin{aligned}
\frac{d V}{d t} \leqslant & 2 \sum_{i=1}^{n} k_{i}\left|f_{i}\left[x_{i}(t)\right]\right|\left[-d_{i}\left|x_{i}(t)\right|+\left|u_{i}\right|\right] \\
& +f^{T}[x(t)]\left[K A+A^{T} K+Q_{1}+Q_{2}+\tau_{2}^{2}\left(1-\eta_{2}\right)^{-1}\right. \\
& \left.\times K C Q_{2}^{-1} C^{T} K+\left(1-\eta_{1}\right)^{-1} K B Q_{1}^{-1} B^{T} K\right] f[x(t)] \\
& \leqslant 2 \sum_{i=1}^{n} k_{i}\left|f_{i}\left[x_{i}(t)\right]\right|\left[-d_{i}\left|x_{i}(t)\right|+\left|u_{i}\right|\right]<0, \\
& \text { when } x \in \mathbb{R}^{n} \backslash \bar{S}_{1} .
\end{aligned}
$$

Hence, when (5) holds, the neural network model in (1) is globally point dissipative and the set $\bar{S}_{1}$ is positively invariant and globally attractive.

(iv) Consider the Lyapunov-Krasovskii functional as in (14). Similar to (15), we have

$$
\begin{aligned}
\frac{d \widetilde{V}}{d t} \leqslant & 2 \sum_{i=1}^{n} \sum_{j=1}^{n} \frac{p_{i}}{\varepsilon}\left|x_{i}(t)\right|\left\{-d_{j}\left|\left[x_{j}(t)\right]\right|+\varepsilon\left|u_{j}\right|\right\} \\
& +2 \sum_{i=1}^{n} k_{i} f_{i}\left[x_{i}(t)\right]\left[-d_{i} x_{i}(t)+u_{i}\right]+\eta^{T}(t) \Omega^{\prime} \eta(t)<0 \\
& \text { when } x \in \mathbb{R}^{n} \backslash \bar{S}_{2},
\end{aligned}
$$

where $\quad \eta^{T}(t)=\left\{x^{T}(t), f^{T}[x(t)], f^{T}\left\{x\left[t-\tau_{1}(t)\right]\right\}, \tau_{2}^{-1} \int_{t-\tau_{2}(t)}^{t}\right.$ $\left.\times f^{T}[x(s)] d s\right\}$ and $\Omega^{\prime}$ is defined as (13). Inequality (17) holds due to the negative definiteness of $\Omega^{\prime}$. Hence, when LMI (5) is satisfied, the neural network model in (1) is globally point dissipative, and the set $\bar{S}_{2}$ is positively invariant and globally attractive. This completes the proof.

In the special case that $c_{i j}=0$, model (2) can be written as the following neural networks with only discrete delays: $\frac{d x_{i}(t)}{d t}=-d_{i} x_{i}(t)+\sum_{j=1}^{n} a_{i j} f_{j}\left[x_{j}(t)\right]+\sum_{j=1}^{n} b_{i j} f_{j}\left\{x_{j}\left[t-\tau_{1}(t)\right]\right\}+u_{i}$.

For model (18), we have the following corollaries which can be obtained easily from Theorem 1 .

Corollary 1: Let assumption A be satisfied with $C=0$. Neural network (18) is a globally point dissipative system if there exist matrices $Q_{1}>0$ and diagonal $K>0$ such that the following LMI holds:

$$
\left[\begin{array}{cc}
K A+A^{T} K+Q_{1} & K B \\
B^{T} K & -\left(1-\eta_{1}\right) Q_{1}
\end{array}\right]<0 .
$$

Moreover, (i), (ii), (iii), and (iv) in Theorem 1 hold.

Corollary 2: Let assumption A be satisfied with $\tau_{1}(t)$ being constant. Neural network (18) is a globally point dissipative system, if the following LMI holds:

$$
\left[\begin{array}{cc}
A+A^{T}+2 I & B \\
B^{T} & -2 I
\end{array}\right]<0 .
$$

Moreover, (i), (ii), (iii), and (iv) in Theorem 1 hold.

Remark 2: Case (i) of Corollary 2 coincides with Theorem 3 in Ref. 27. Hence, our result improves and generalizes an earlier result.

\section{B. Results using Lyapunov functions}

In this section, conditions are given for global point dissipativity of (1) by constructing appropriate Lyapunov functions and employing different bounding techniques.

Theorem 2: Let $f(x) \in \mathcal{B}$ and Assumption A be satisfied. Neural network (1) is globally point dissipative, and the set $\mathcal{E}=\left\{x|| x_{i} \mid \leqslant M_{i}\right\}$, where $M_{i} \triangleq \sum_{j=1}^{n} d_{i}^{-1}\left[\left(\left|a_{i j}\right|+\left|b_{i j}\right|+\tau_{2}\left|c_{i j}\right|\right) h_{j}\right.$ $\left.+\left|u_{i}\right|\right]$, is positively invariant and globally attractive.

Proof: Construct a radially unbounded and positive definite Lyapunov function candidate as 


$$
V(x)=\sum_{i=1}^{n} \frac{\left|x_{i}\right|^{r}}{r} .
$$

By computing $D^{+} V$ along the trajectory of (1), we have

$$
\begin{aligned}
\left.D^{+} V(x)\right|_{(1)} & =\sum_{i=1}^{n}\left|x_{i}\right|^{r-1} \operatorname{sgn}\left(x_{i}\right) \frac{d x_{i}}{d t} \\
& =\sum_{i=1}^{n}\left|x_{i}\right|^{r-1} \operatorname{sgn}\left(x_{i}\right)\left[-d_{i} x_{i}(t)+\sum_{j=1}^{n} a_{i j} f_{j}\left[x_{j}(t)\right]+\sum_{j=1}^{n} b_{i j} f_{j}\left[x_{j}\left[t-\tau_{1}(t)\right]\right\}+\sum_{j=1}^{n} c_{i j} \int_{t-\tau_{2}(t)}^{t} f_{j}\left[x_{j}(s)\right] d s+u_{i}\right] \\
& \leqslant \sum_{i=1}^{n}\left[-d_{i}\left|x_{i}\right|^{r}+\sum_{j=1}^{n}\left(\left|a_{i j}\right|+\left|b_{i j}\right|+\tau_{2}\left|c_{i j}\right|\right) h_{j}\left|x_{i}\right|^{r-1}+\left|u_{i}\right|\left|x_{i}\right|^{r-1}\right] \\
& =-\sum_{i=1}^{n} d_{i}\left|x_{i}\right|^{r}+\sum_{i=1}^{n} \sum_{j=1}^{n}\left[\left(\left|a_{i j}\right|+\left|b_{i j}\right|+\tau_{2}\left|c_{i j}\right|\right) h_{j}+\left|u_{i}\right|\right]\left|x_{i}\right|^{r-1} \\
& =-\sum_{i=1}^{n}\left|x_{i}\right|^{r-1}\left[d_{i}\left|x_{i}\right|-\sum_{j=1}^{n}\left[\left(\left|a_{i j}\right|+\left|b_{i j}\right|+\tau_{2}\left|c_{i j}\right|\right) h_{j}+\left|u_{i}\right|\right]\right]<0,
\end{aligned}
$$

when $x \in \mathbb{R}^{n} \backslash \mathcal{E}$, where

$$
\mathcal{E}=\left\{x|| x_{i} \mid \leqslant d_{i}^{-1} \sum_{j=1}^{n}\left[\left(\left|a_{i j}\right|+\left|b_{i j}\right|+\tau_{2}\left|c_{i j}\right|\right) h_{j}+\left|u_{i}\right|\right]\right\} .
$$

From Lemma 4, the neural network (1) is point dissipative. (20) implies that $\forall x_{0} \in \mathcal{E}, x\left(t, t_{0}, x_{0}\right) \subseteq \mathcal{E}$ holds, $t \geqslant t_{0}$. For $x_{0} \notin \mathcal{E}$, there exists $T\left(x_{0}\right)>0$ such that $x\left(t, t_{0}, x_{0}\right) \subseteq \mathcal{E}, t$ $\geqslant T\left(x_{0}\right)+t_{0}$, therefore $\mathcal{E}$ is positively invariant and attractive. The proof is completed.

Theorem 3: Let $f(x) \in \mathcal{B}$ and Assumption A be satisfied. Neural network (1) is globally exponentially point dissipative, and the set $\mathcal{E}^{*}=\left\{x|| x_{i} \mid \leqslant d_{i} M_{i} /\left(d_{i}-\varepsilon\right)\right\}$, where $\varepsilon>0$ is a sufficiently small constant, is positively invariant and globally exponentially attractive.

Proof: Obviously, $\mathcal{E}^{*}$ is a positive invariant set because $\mathcal{E}^{*} \subset \mathcal{E}$. Now we consider a Lyapunov function candidate

$$
V(x)=\sum_{i=1}^{n} \frac{e^{\varepsilon t}\left|x_{i}\right|^{r}}{r} .
$$

By calculating the $D^{+} V$ along the solution of the model (1) and by (20), we have

$$
\begin{aligned}
\left.D^{+} V[x(t)]\right|_{(2)} & =\sum_{i=1}^{n}\left[e^{\varepsilon t} \frac{d}{d t}\left(\frac{\left|x_{i}\right|^{r}}{r}\right)+\varepsilon e^{\varepsilon t} \frac{\left|x_{i}\right|^{r}}{r}\right] \\
& \leqslant \frac{e^{\varepsilon t}}{r} \sum_{i=1}^{n}\left|x_{i}\right|^{r-1}\left[\left(\varepsilon-d_{i}\right)\left|x_{i}\right|+d_{i} M_{i}\right]<0,
\end{aligned}
$$

when $\left|x_{i}\right|>d_{i} M_{i} /\left(d_{i}-\varepsilon\right)$, that is, $x \in \mathbb{R}^{n} \backslash \mathcal{E}^{*}$. Integrating two sides of (21) from $t_{0}$ to an arbitrary $t>t_{0}$, we have $V[x(t)]$ $\leqslant V\left[x\left(t_{0}\right)\right]$. Therefore, we have

$$
\sum_{i=1}^{n}\left|x_{i}(t)\right|^{r} \leqslant e^{-\varepsilon\left(t-t_{0}\right)} \sum_{i=1}^{n}\left|x_{i}\left(t_{0}\right)\right|^{r} .
$$

It follows that

$$
\begin{aligned}
& \inf _{x_{0} \in \mathbb{R}^{n} \backslash \mathcal{E}^{*}}\left\{\left\|x\left(t, t_{0}, x_{0}\right)-\widetilde{x}\right\|_{r} \mid \widetilde{x} \in \mathcal{E}^{*}\right\} \\
& \quad \leqslant\left\|x\left(t, t_{0}, x_{0}\right)\right\|_{r} \\
& \quad=\left(\sum_{i=1}^{n}\left|x_{i}\left(t, t_{0}, x_{0}\right)\right|^{r}\right)^{1 / r} \\
& \quad \leqslant e^{-\varepsilon\left(t-t_{0}\right) / r}\left(\sum_{i=1}^{n}\left|x_{i}\left(t_{0}\right)\right|^{r}\right)^{1 / r} .
\end{aligned}
$$

If we take $M\left(x_{0}\right)=\left\|x_{0}\right\|_{r}$, we have

$$
\inf _{x_{0} \in \mathrm{R}^{n} \backslash \mathcal{E}^{*}}\left\{\left\|x\left(t, t_{0}, x_{0}\right)-\widetilde{x}\right\|_{r} \mid \widetilde{x} \in \mathcal{E}^{*}\right\} \leqslant M\left(x_{0}\right) e^{-\varepsilon\left(t-t_{0}\right) / r} .
$$

The inequality in (22) means that the set $\mathcal{E}^{*}$ is globally exponentially attractive, that is, the models (1) or (2) are globally exponentially point dissipative. This completes the proof.

\section{ILLUSTRATIVE EXAMPLES}

Example 1: Consider the following neural network model with discrete time delay and distributed delay:

$$
\begin{aligned}
\frac{d x(t)}{d t}= & -D x(t)+A f[x(t)]+B f\left\{x\left[t-\tau_{1}(t)\right]\right\} \\
& +C \int_{t-\tau_{2}(t)}^{t} f[x(s)] d s+u,
\end{aligned}
$$

where $f_{i}\left(x_{i}\right)=\arctan \left(x_{i}\right)$ and 


$$
\begin{aligned}
& D=\left[\begin{array}{ll}
1 & 0 \\
0 & 1
\end{array}\right], \quad A=\left[\begin{array}{cc}
-0.9 & 0 \\
0 & -0.9
\end{array}\right], \\
& B=\left[\begin{array}{cc}
0 & -0.02 \\
0.02 & -0.06
\end{array}\right], \quad C=\left[\begin{array}{cc}
0.10 & -0.02 \\
-0.26 & 0.1
\end{array}\right], \\
& u=\left[\begin{array}{l}
1 \\
1
\end{array}\right] .
\end{aligned}
$$

For notational and computational convenience, let $\tau_{1}(t)$ $=\tau_{2}(t)=1$, then $\eta_{1}=\eta_{2}=0$. Take $K=I$ and $Q_{1}=Q_{2}=\frac{1}{2} I$, the matrix inequality (5) in Theorem 1 holds, that is,

$$
\begin{aligned}
{\left[\begin{array}{ccc}
A+A^{T}+Q_{1}+Q_{2} & B & C \\
B^{T} & -Q_{1} & 0 \\
C^{T} & 0 & -Q_{2}
\end{array}\right] } \\
\quad=\left[\begin{array}{cccccc}
-0.8 & 0 & 0 & -0.02 & 0.1 & -0.02 \\
0 & -0.8 & 0.02 & -0.06 & -0.26 & 0.1 \\
0 & 0.02 & -0.5 & 0 & 0 & 0 \\
-0.02 & -0.06 & 0 & -0.5 & 0 & 0 \\
0.1 & -0.26 & 0 & 0 & -0.5 & 0 \\
-0.02 & 0.1 & 0 & 0 & 0 & -0.5
\end{array}\right]<0 .
\end{aligned}
$$

From Theorem 1, the model in (23) is globally point dissipative. Note that $l_{i}=1$ and the activation functions $f_{i}(\cdot) \in \mathcal{L}$, thus the globally attractive set is $S_{1}=\left\{x|| \arctan \left(x_{i}\right) \mid\right.$ $\left.\leqslant l_{i}\left|u_{i}\right| / d_{i}=1, i=1,2, \ldots, n\right\}$; also the activation functions $f_{i}(\cdot) \in \mathcal{G}$, then the globally attractive set is $\bar{S}_{1}=\left\{x|| x_{i} \mid\right.$ $\left.\leqslant\left|u_{i}\right| / d_{i}=1, i=1,2, \ldots, n\right\}$. To compare the results in Ref. 27 let $C=0$ in model (23), and then consider the neural network model with only discrete time delay. Take $K=I$ and $Q_{1}=\frac{1}{2} I$, then the linear matrix inequality in Corollary 1 holds, that is,

$$
\begin{aligned}
& {\left[\begin{array}{cc}
A+A^{T}+Q_{1} & B \\
B^{T} & -Q_{1}
\end{array}\right]} \\
& =\left[\begin{array}{cccc}
-1.3 & 0 & 0 & -0.02 \\
0 & -1.3 & 0.02 & -0.06 \\
0 & 0.02 & -0.5 & 0 \\
-0.02 & -0.06 & 0 & -0.5
\end{array}\right]<0 .
\end{aligned}
$$

By Corollary 1, we conclude that the model with only discrete time delays is a globally point dissipative system. However,

$$
\left[\begin{array}{cc}
A+A^{T}+2 I & B \\
B^{T} & -2 I
\end{array}\right]=\left[\begin{array}{cccc}
0.2 & 0 & 0 & -0.02 \\
0 & 0.2 & 0.02 & -0.06 \\
0 & 0.02 & -2 & 0 \\
-0.02 & -0.06 & 0 & -2
\end{array}\right]
$$

is not negative definite; hence the criterion given in Ref. 27 is not applicable. This implies that the proposed results improve and generalize those in Ref. 27.

Note that $\left|\arctan \left(x_{i}\right)\right| \leqslant \pi / 2$, hence the activations $f_{i}\left(x_{i}\right)$ $=\arctan \left(x_{i}\right) \in \mathcal{B}$. By virtue of Theorem 2, we have $M_{1}$ $=0.52 \pi+1$ and $M_{2}=0.67 \pi+1$, the globally attractive set is $\mathcal{E}=\left\{x=\left(x_{1}, x_{2}\right)^{T}|| x_{1}|\leqslant 0.52 \pi+1,| x_{2} \mid \leqslant 0.67 \pi+1\right\}$.

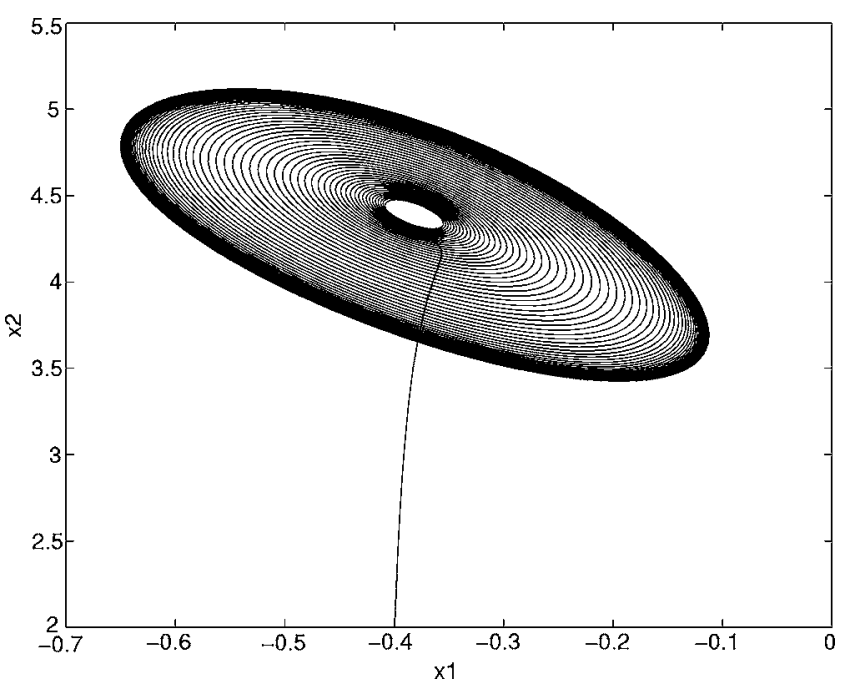

FIG. 1. Transient response of state variables $x_{1}(t)$ and $x_{2}(t)$.

The following example is given to illustrate that when the sufficient condition ensuring the global point dissipativity is not satisfied, the complex dynamics will appear.

Example 2: Consider the following neural network model with discrete time delay:

$$
\frac{d x(t)}{d t}=-D x(t)+A f[x(t)]+B f\left\{x\left[t-\tau_{1}(t)\right]\right\}+u,
$$

where $f_{i}\left(x_{i}\right)=\tanh \left(x_{i}\right),(i=1,2), \tau_{1}(t)=1$, and $D=\left[\begin{array}{ll}1 & 0 \\ 0 & 1\end{array}\right]$.

In Corollary 1 , if

$$
A=\left[\begin{array}{cc}
2 & -0.1 \\
-5 & 4.5
\end{array}\right], \quad B=\left[\begin{array}{cc}
-1.5 & -0.1 \\
-0.2 & -3
\end{array}\right],
$$

the linear matrix inequality (19) does not have a feasible solution, in other words, the system (24) is not a dissipative system. Figure 1 depicts the states of system (24) with initial conditions $x_{1}(t) \equiv-0.4, x_{2}(t) \equiv 2, t \in[-1,0]$, and input vectors $u=\left[\begin{array}{l}0 \\ 1\end{array}\right]$.

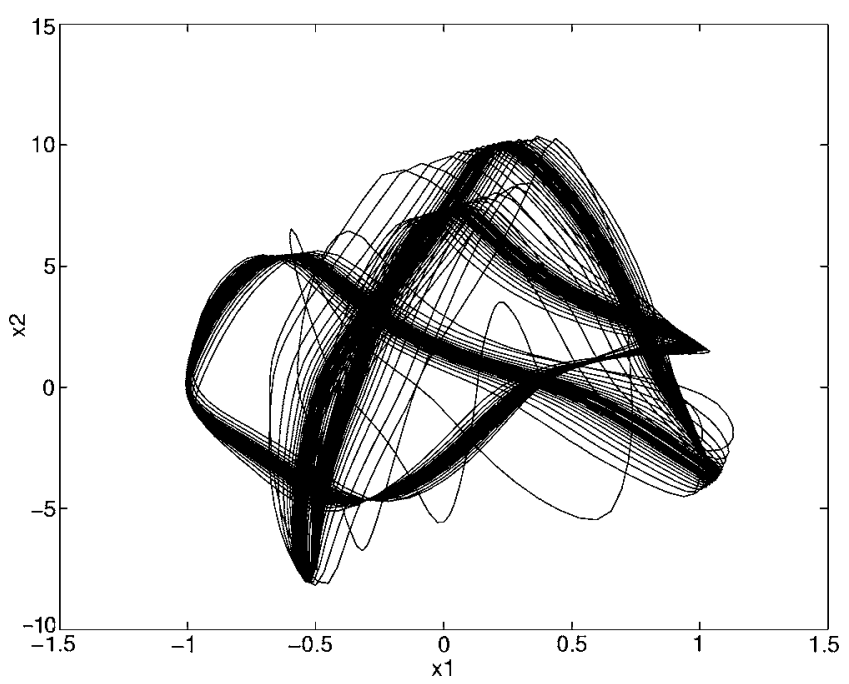

FIG. 2. Transient response of state variables $x_{1}(t)$ and $x_{2}(t)$. 


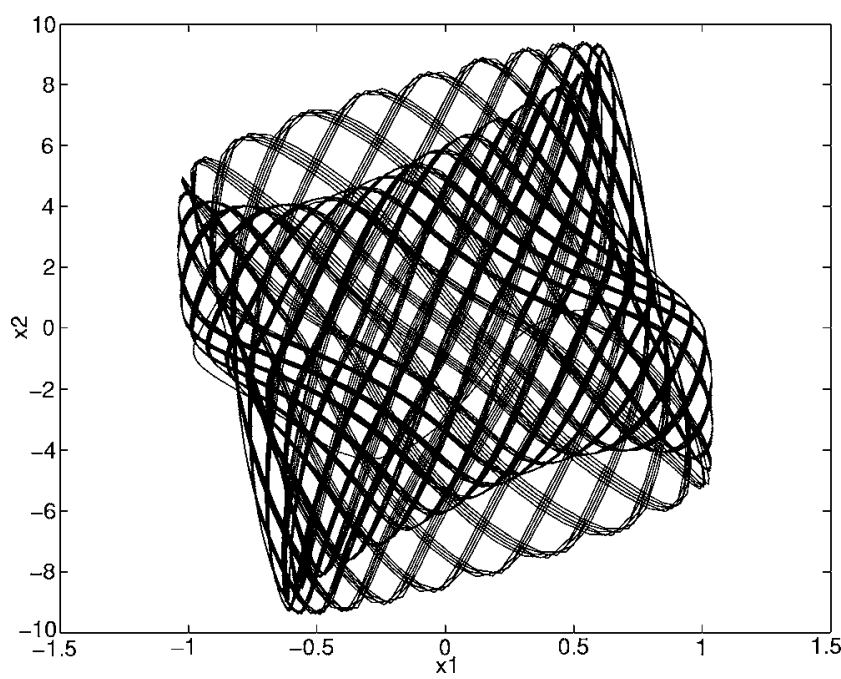

FIG. 3. Transient response of state variables $x_{1}(t)$ and $x_{2}(t)$.

Similarly, in Corollary 1, if

$$
A=\left[\begin{array}{ll}
2 & 0.1 \\
5 & 4.5
\end{array}\right], \quad B=\left[\begin{array}{cc}
-1.5 & 0.1 \\
0.2 & -8.5
\end{array}\right],
$$

the linear matrix inequality (19) does not have a feasible solution, in other words, the system (24) is not a dissipative system. Here, Fig. 2 depicts the states of system (24) with initial conditions $x_{1}(t) \equiv 0.4, x_{2}(t) \equiv 0.2, t \in[-1,0]$, and input vectors $u=\left[\begin{array}{c}0 \\ 1.5\end{array}\right]$; Fig. 3 depicts the states of system (24) with initial conditions $x_{1}(t) \equiv 0.6, x_{2}(t) \equiv 0.6, t \in[-1,0]$, and zero input vectors.

\section{CONCLUSIONS}

In this paper, we have studied the global point dissipativity of neural networks with both discrete time-varying delays and distributed time-varying delays. Using Lyapunov methods and LMI techniques, several new sufficient conditions and globally attractive sets have been derived for the point dissipativity of neural networks with mixed timevarying delays. The advantage of the proposed approach is that the developed criteria can be performed efficiently via numerical algorithms such as interior-point algorithms for solving LMIs. The new results given in this paper generalize and improve the earlier ones, and the new conditions are easy to check and apply in practice. Illustrated examples are also given to demonstrate the effectiveness of the proposed results.

\section{ACKNOWLEDGMENTS}

This work was supported by the National Natural Science Foundation of China under Grants No. 60574043 and No. 60373067, the Natural Science Foundation of Jiangsu Province under Grant No. BK2003053, and the HKU CRCG Grant No. 10205878.
${ }^{1}$ T. Roska, C. W. Wu, and L. O. Chua, "Stability of cellular neural networks with dominant nonlinear and delay-type template," IEEE Trans. Circuits Syst., I: Fundam. Theory Appl. 40, 270-272 (1993).

${ }^{2} \mathrm{~N}$. Takahashi, "A new sufficient for complete stability of cellular neural networks with delay," IEEE Trans. Circuits Syst., I: Fundam. Theory Appl. 47, 793-799 (2000).

${ }^{3}$ J. Cao and J. Wang, "Global asymptotic and robust stability of recurrent neural networks with time delays," IEEE Trans. Circuits Syst., I: Fundam. Theory Appl. 52, 417-426 (2005).

${ }^{4}$ J. Cao and L. Wang, "Exponential stability and periodic oscillatory solution in BAM networks with delays," IEEE Trans. Neural Netw. 13, 457463 (2002).

${ }^{5}$ J. Cao, "Global stability conditions for delayed CNNs," IEEE Trans. Circuits Syst., I: Fundam. Theory Appl. 48, 1330-1333 (2001).

${ }^{6} \mathrm{M}$. Gilli, "Stability of cellular neural networks and delayed cellular neural networks with nonpositive templates and nonmonotonic output functions," IEEE Trans. Circuits Syst., I: Fundam. Theory Appl. 41, 518-528 (1994). ${ }^{7}$ A. Chen, J. Cao, and L. Huang, "An estimation of upper bound of delays for global asymptotic stability of delayed Hopfield neural networks," IEEE Trans. Circuits Syst., I: Fundam. Theory Appl. 49, 1028-1032 (2000).

${ }^{8} \mathrm{~S}$. Arik, "An analysis of global asymptotic stability of delayed cellular neural networks," IEEE Trans. Neural Netw. 13, 1239-1242 (2002).

${ }^{9} \mathrm{~T}$. Chen, "Global exponential stability of delayed Hopfield neural networks," Neural Networks 14, 977-980 (2001).

${ }^{10} \mathrm{C}$. M. Marcus and R. M. Westervelt, "Stability of analog neural networks with delays," Phys. Rev. A 39, 347-359 (1989).

${ }^{11}$ P. V. D. Driessche and X. Zou, "Global attractivity in delayed Hopfield neural network models," SIAM J. Appl. Math. 58, 1878-1890 (1998).

${ }^{12} \mathrm{C}$. Hou and J. Qian, "Stability analysis for neural dynamics with timevarying delays," IEEE Trans. Neural Netw. 9, 221-223 (1998).

${ }^{13} \mathrm{~K}$. Gopalsamy and X. Z. He, "Delay-independent stability in bidirectional associative memory networks," IEEE Trans. Neural Netw. 5, 998-1002 (1994).

${ }^{14} \mathrm{C}$. Feng and R. Plamondon, "On the stability analysis of delayed neural networks systems," Neural Networks 14, 1181-1188 (2001).

${ }^{15}$ T. Liao and F. Wang, "Global stability for cellular neural networks with time delay," IEEE Trans. Neural Netw. 11, 1481-1484 (2000).

${ }^{16} \mathrm{X}$. Liang and J. Wang, "An additive diagonal-stability condition for absolute exponential stability of a general class of neural networks," IEEE Trans. Circuits Syst., I: Fundam. Theory Appl. 48, 1308-1317 (2001).

${ }^{17} \mathrm{~J}$. Cao and J. Wang, "Absolute exponential stability of recurrent neural networks with Lipschitz-continuous activation functions and time delays," Neural Networks 17, 379-390 (2004).

${ }^{18}$ M. Forti, S. Manetti, and M. Marini, "Necessary and sufficient condition for absolute stability of neural networks," IEEE Trans. Circuits Syst., I: Fundam. Theory Appl. 41, 491-494 (1994).

${ }^{19}$ Y. Zhang, P. Heng, and P. Vadakkepat, "Absolute periodicity and absolute stability of delayed neural networks," IEEE Trans. Circuits Syst., I: Fundam. Theory Appl. 49, 256-261 (2002).

${ }^{20} \mathrm{Z}$. Xu, H. Qiao, J. Peng, and B. Zhang, "A comparative study of two modeling approaches in neural networks," Neural Networks 17, 73-85 (2004).

${ }^{21}$ J. C. Principle, J. M. Kuo, and S. Celebi, "An analysis of the gamma memory in dynamic neural networks," IEEE Trans. Neural Netw. 5, 331337 (1994).

${ }^{22}$ S. Ruan and R. S. Filfil, "Dynamics of a two-neuron system with discrete and distributed delays," Physica D 191, 323-342 (2004).

${ }^{23}$ X. Liao, K. Wong, and S. Yang, "Convergence dynamics of hybrid bidirectional associative memory neural networks with distributed delays," Phys. Lett. A 316, 55-64 (2003).

${ }^{24} \mathrm{Q}$. Zhang, X. Wei, and J. Xu, "Global exponential stability of Hopfield neural networks with continuously distributed delays," Phys. Lett. A 315, 431-436 (2003)

${ }^{25} \mathrm{H}$. Zhao, "Global asymptotic stability of Hopfield neural network involving distributed delays," Neural Networks 17, 47-53 (2004).

${ }^{26}$ Z. Wang, Y. Liu, and X. Liu, "On global asymptotic stability of neural networks with discrete and distributed delays," Phys. Lett. A 345, 299308 (2005).

${ }^{27} \mathrm{X}$. Liao and J. Wang, "Global dissipativity of continuous-time recurrent neural networks with time delay,” Phys. Rev. E 68, 016118 (2003).

${ }^{28}$ X. Liao, Y. Fu, and Y. Guo, "Partial dissipative property for a class of nonlinear systems with separated variables," J. Math. Anal. Appl. 173, 103-115 (1993).

${ }^{29} \mathrm{Q}$. Kong and X. Liao, "Dissipation, boundedness and persistence of general ecological systems," Nonlinear Anal. Theory, Methods Appl. 25, 
1237-1250 (1995).

${ }^{30} \mathrm{~S}$. Xu, T. Chen, and J. Lam, "Robust $H_{\infty}$ filtering for uncertain Markovian jump systems with mode-dependent time delays," IEEE Trans. Autom. Control 48, 900-907 (2003).

${ }^{31}$ S. Xu, J. Lam, Daniel W. C. Ho, and Y. Zou, "Global robust exponential stability analysis for interval recurrent neural networks," Phys. Lett. A 325, 124-133 (2004).
${ }^{32}$ S. Boyd, L. E. Ghaoui, E. Feron, and V. Balakrishnan, Linear Matrix Inequalities in System and Control Theory (SIAM, Philadelphia, PA, 1994).

${ }^{33} \mathrm{~K}$. Gu, V. Kharitonov, and J. Chen, Stability of Time-Delay Systems (Birkhauser, Boston, MA, 2003).

${ }^{34}$ J. Hale, Asymptotic Behavior of Dissipative Systems (American Mathematical Society, New York, 1989). 